\title{
Schur-convexity of dual form of some symmetric functions
}

\author{
Huan-Nan Shi ${ }^{1}$ and Jing Zhang ${ }^{2^{*}}$
}

\section{*Correspondence:}

Idtzhangjing1@buu.edu.cn

${ }^{2}$ Basic Courses Department, Beijing

Union University, Beijing, 100101,

P.R. China

Full list of author information is

available at the end of the article

\begin{abstract}
By the properties of a Schur-convex function, Schur-convexity of the dual form of some symmetric functions is simply proved.

MSC: Primary 26D15; 05E05; 26B25

Keywords: majorization; Schur-convexity; inequality; symmetric functions; dual form; convex function
\end{abstract}

\section{Introduction}

Throughout the article, $\mathbb{R}$ denotes the set of real numbers, $\boldsymbol{x}=\left(x_{1}, x_{2}, \ldots, x_{n}\right)$ denotes $n$-tuple ( $n$-dimensional real vectors), the set of vectors can be written as

$$
\begin{aligned}
& \mathbb{R}^{n}=\left\{\boldsymbol{x}=\left(x_{1}, \ldots, x_{n}\right): x_{i} \in \mathbb{R}, i=1, \ldots, n\right\}, \\
& \mathbb{R}_{+}^{n}=\left\{\boldsymbol{x}=\left(x_{1}, \ldots, x_{n}\right): x_{i}>0, i=1, \ldots, n\right\} .
\end{aligned}
$$

In particular, the notations $\mathbb{R}$ and $\mathbb{R}_{+}$denote $\mathbb{R}^{1}$ and $\mathbb{R}_{+}^{1}$, respectively.

For convenience, we introduce some definitions as follows.

Definition $1[1,2]$ Let $\boldsymbol{x}=\left(x_{1}, \ldots, x_{n}\right)$ and $\boldsymbol{y}=\left(y_{1}, \ldots, y_{n}\right) \in \mathbb{R}^{n}$.

(i) $\boldsymbol{x} \geq \boldsymbol{y}$ means $x_{i} \geq y_{i}$ for all $i=1,2, \ldots, n$.

(ii) Let $\Omega \subset \mathbb{R}^{n}, \varphi: \Omega \rightarrow \mathbb{R}$ is said to be increasing if $\boldsymbol{x} \geq \boldsymbol{y}$ implies $\varphi(\boldsymbol{x}) \geq \varphi(\boldsymbol{y}) . \varphi$ is said to be decreasing if and only if $-\varphi$ is increasing.

Definition $2[1,2]$ Let $\boldsymbol{x}=\left(x_{1}, \ldots, x_{n}\right)$ and $\boldsymbol{y}=\left(y_{1}, \ldots, y_{n}\right) \in \mathbb{R}^{n}$.

(i) $\boldsymbol{x}$ is said to be majorized by $\boldsymbol{y}$ (in symbols $\boldsymbol{x} \prec \boldsymbol{y}$ ) if $\sum_{i=1}^{k} x_{[i]} \leq \sum_{i=1}^{k} y_{[i]}$ for $k=1,2, \ldots, n-1$ and $\sum_{i=1}^{n} x_{i}=\sum_{i=1}^{n} y_{i}$, where $x_{[1]} \geq \cdots \geq x_{[n]}$ and $y_{[1]} \geq \cdots \geq y_{[n]}$ are rearrangements of $\boldsymbol{x}$ and $\boldsymbol{y}$ in a descending order.

(ii) Let $\Omega \subset \mathbb{R}^{n}, \varphi: \Omega \rightarrow \mathbb{R}$ is said to be a Schur-convex function on $\Omega$ if $\boldsymbol{x} \prec \boldsymbol{y}$ on $\Omega$ implies $\varphi(\boldsymbol{x}) \leq \varphi(\boldsymbol{y}) . \varphi$ is said to be a Schur-concave function on $\Omega$ if and only if $-\varphi$ is Schur-convex function on $\Omega$.

Definition $3[1,2]$ Let $\boldsymbol{x}=\left(x_{1}, \ldots, x_{n}\right)$ and $\boldsymbol{y}=\left(y_{1}, \ldots, y_{n}\right) \in \mathbb{R}^{n}$.

(i) $\Omega \subset \mathbb{R}^{n}$ is said to be a convex set if $\boldsymbol{x}, \boldsymbol{y} \in \Omega, 0 \leq \alpha \leq 1$ implies $\alpha \mathbf{X}+(1-\alpha) \boldsymbol{y}=\left(\alpha x_{1}+(1-\alpha) y_{1}, \ldots, \alpha x_{n}+(1-\alpha) y_{n}\right) \in \Omega$. 
(ii) Let $\Omega \subset \mathbb{R}^{n}$ be a convex set. A function $\varphi: \Omega \rightarrow \mathbb{R}$ is said to be a convex function on $\Omega$ if

$$
\varphi(\alpha \mathbf{x}+(1-\alpha) \mathbf{y}) \leq \alpha \varphi(\mathbf{x})+(1-\alpha) \varphi(\boldsymbol{y})
$$

for all $\boldsymbol{x}, \boldsymbol{y} \in \Omega$ and all $\alpha \in[0,1] . \varphi$ is said to be a concave function on $\Omega$ if and only if $-\varphi$ is a convex function on $\Omega$.

(iii) Let $\Omega \subset \mathbb{R}^{n}$. A function $\varphi: \Omega \rightarrow \mathbb{R}$ is said to be a log-convex function on $\Omega$ if the function $\ln \varphi$ is convex.

\section{Definition 4 [1]}

(i) $\Omega \subset \mathbb{R}^{n}$ is called a symmetric set, if $x \in \Omega$ implies $P x \in \Omega$ for every $n \times n$ permutation matrix $P$.

(ii) The function $\varphi: \Omega \rightarrow \mathbb{R}$ is called symmetric if for every permutation matrix $P$, $\varphi(P x)=\varphi(x)$ for all $x \in \Omega$.

Theorem A (Schur-convex function decision theorem [1, p.84]) Let $\Omega \subset \mathbb{R}^{n}$ be symmetric and have a nonempty interior convex set. $\Omega^{0}$ is the interior of $\Omega . \varphi: \Omega \rightarrow \mathbb{R}$ is continuous on $\Omega$ and differentiable in $\Omega^{0}$. Then $\varphi$ is the Schur-convex (Schur-concave) function if and only if $\varphi$ is symmetric on $\Omega$ and

$$
\left(x_{1}-x_{2}\right)\left(\frac{\partial \varphi}{\partial x_{1}}-\frac{\partial \varphi}{\partial x_{2}}\right) \geq 0(\leq 0)
$$

holds for any $\mathbf{x} \in \Omega^{0}$.

The Schur-convex functions were introduced by Schur in 1923 and have important applications in analytic inequalities, elementary quantum mechanics and quantum information theory. See [1].

In recent years, many scholars use the Schur-convex function decision theorem to determine the Schur-convexity of many symmetric functions.

Xia et al. [3] proved that the symmetric function

$$
E_{k}\left(\frac{\boldsymbol{x}}{1+\boldsymbol{x}}\right)=\sum_{1 \leq i_{1}<\cdots<i_{k} \leq n} \prod_{j=1}^{k} \frac{x_{i_{j}}}{1+x_{i_{j}}}, \quad k=1, \ldots, n,
$$

is Schur-convex on $\mathbb{R}_{+}^{n}$.

Chu et al. [4] proved that the symmetric function

$$
E_{k}\left(\frac{\boldsymbol{x}}{1-\boldsymbol{x}}\right)=\sum_{1 \leq i_{1}<\cdots<i_{k} \leq n} \prod_{j=1}^{k} \frac{x_{i_{j}}}{1-x_{i_{j}}}, \quad k=1, \ldots, n,
$$

is Schur-convex on $\left[\frac{k-1}{2(n-1)}, 1\right)^{n}$ and Schur-concave on $\left[0, \frac{k-1}{2(n-1)}\right]^{n}$.

$\mathrm{Xia}$ and Chu [5] proved that the symmetric function

$$
E_{k}\left(\frac{1-\boldsymbol{x}}{\boldsymbol{x}}\right)=\sum_{1 \leq i_{1}<\cdots<i_{k} \leq n} \prod_{j=1}^{k} \frac{1-x_{i_{j}}}{x_{i_{j}}}, \quad k=1, \ldots, n,
$$

is Schur-convex on $\left(0, \frac{2 n-k-1}{2(n-1)}\right]^{n}$ and Schur-concave on $\left[\frac{2 n-k-1}{2(n-1)}, 1\right]^{n}$. 
Xia and Chu [6] also proved that the symmetric function

$$
E_{k}\left(\frac{1+\boldsymbol{x}}{1-\boldsymbol{x}}\right)=\sum_{1 \leq i_{1}<\cdots<i_{k} \leq n} \prod_{j=1}^{k} \frac{1+x_{i_{j}}}{1-x_{i_{j}}}, \quad k=1, \ldots, n,
$$

is Schur-convex on $(0,1)^{n}$.

Mei et al. [7] proved that the symmetric function

$$
E_{k}\left(\frac{1}{\boldsymbol{x}}-\boldsymbol{x}\right)=\sum_{1 \leq i_{1}<\cdots<i_{k} \leq n} \prod_{j=1}^{k}\left(\frac{1}{x_{i_{j}}}-x_{i_{j}}\right), \quad k=1, \ldots, n,
$$

is Schur-convex on $(0,1)^{n}$. More results for Schur convexity of the symmetric functions, we refer the reader to [8].

In this paper, by the properties of a Schur-convex function, we study Schur-convexity of the dual form of the above symmetric functions, and we obtained the following results.

Theorem 1 The symmetric function

$$
E_{k}^{*}\left(\frac{\boldsymbol{x}}{1+\boldsymbol{x}}\right)=\prod_{1 \leq i_{1}<\cdots<i_{k} \leq n} \sum_{j=1}^{k} \frac{x_{i_{j}}}{1+x_{i_{j}}}, \quad k=1, \ldots, n,
$$

is a Schur-concave function on $\mathbb{R}_{+}^{n}$.

Theorem 2 The symmetric function

$$
E_{k}^{*}\left(\frac{\boldsymbol{x}}{1-\boldsymbol{x}}\right)=\prod_{1 \leq i_{1}<\cdots<i_{k} \leq n} \sum_{j=1}^{k} \frac{x_{i_{j}}}{1-x_{i_{j}}}, \quad k=1, \ldots, n,
$$

is a Schur-convex function on $\left[\frac{1}{2}, 1\right)^{n}$.

Theorem 3 The symmetric function

$$
E_{k}^{*}\left(\frac{1-\boldsymbol{x}}{\boldsymbol{x}}\right)=\prod_{1 \leq i_{1}<\cdots<i_{k} \leq n} \sum_{j=1}^{k} \frac{1-x_{i_{j}}}{x_{i_{j}}}, \quad k=1, \ldots, n,
$$

is a Schur-convex function on $\left(0, \frac{1}{2}\right]^{n}$.

Theorem 4 The symmetric function

$$
E_{k}^{*}\left(\frac{1+\boldsymbol{x}}{1-\boldsymbol{x}}\right)=\prod_{1 \leq i_{1}<\cdots<i_{k} \leq n} \sum_{j=1}^{k} \frac{1+x_{i_{j}}}{1-x_{i_{j}}}, \quad k=1, \ldots, n,
$$

is a Schur-convex function on $(0,1)^{n}$.

Theorem 5 The symmetric function

$$
E_{k}^{*}\left(\frac{1}{\boldsymbol{x}}-\boldsymbol{x}\right)=\prod_{1 \leq i_{1}<\cdots<i_{k} \leq n} \sum_{j=1}^{k}\left(\frac{1}{x_{i_{j}}}-x_{i_{j}}\right), \quad k=1, \ldots, n,
$$

is a Schur-convex function on $(0, \sqrt{\sqrt{5}-2})^{n}$. 


\section{Lemmas}

To prove the above three theorems, we need the following lemmas.

Lemma 1 ([1, p.97], [2]) If $\varphi$ is symmetric and convex (concave) on a symmetric convex set $\Omega$, then $\varphi$ is Schur-convex (Schur-concave) on $\Omega$.

Lemma 2 [2, p.64] Let $\Omega \subset \mathbb{R}^{n}, \varphi: \Omega \rightarrow \mathbb{R}_{+}$. Then $\log \varphi$ is Schur-convex (Schur-concave) if and only if $\varphi$ is Schur-convex (Schur-concave).

Lemma 3 ([1, p.642], [2]) Let $\Omega \subset \mathbb{R}^{n}$ be an open convex set, $\varphi: \Omega \rightarrow \mathbb{R}$. For $\boldsymbol{x}, \boldsymbol{y} \in \Omega$, define one variable function $g(t)=\varphi(t \boldsymbol{x}+(1-t) \boldsymbol{y})$ on the interval $(0,1)$. Then $\varphi$ is convex (concave) on $\Omega$ if and only if $g$ is convex (concave) on $[0,1]$ for all $\boldsymbol{x}, \boldsymbol{y} \in \Omega$.

Lemma 4 Let $\mathbf{x}=\left(x_{1}, \ldots, x_{m}\right)$ and $\boldsymbol{y}=\left(y_{1}, \ldots, y_{m}\right) \in \mathbb{R}_{+}^{m}$. Then the function $p(t)=\log g(t)$ is concave on $[0,1]$, where

$$
g(t)=\sum_{j=1}^{m} \frac{t x_{j}+(1-t) y_{j}}{1+t x_{j}+(1-t) y_{j}} .
$$

Proof

$$
p^{\prime}(t)=\frac{g^{\prime}(t)}{g(t)}
$$

where

$$
\begin{aligned}
& g^{\prime}(t)=\sum_{j=1}^{m} \frac{x_{j}-y_{j}}{\left(1+t x_{j}+(1-t) y_{j}\right)^{2}}, \\
& p^{\prime \prime}(t)=\frac{g^{\prime \prime}(t) g(t)-\left(g^{\prime}(t)\right)^{2}}{g^{2}(t)},
\end{aligned}
$$

where

$$
g^{\prime \prime}(t)=-\sum_{j=1}^{m} \frac{2\left(x_{j}-y_{j}\right)^{2}}{\left(1+t x_{j}+(1-t) y_{j}\right)^{3}} .
$$

Thus,

$$
\begin{aligned}
g^{\prime \prime}(t) g(t)-\left(g^{\prime}(t)\right)^{2} & \\
= & \left(-\sum_{j=1}^{m} \frac{2\left(x_{j}-y_{j}\right)^{2}}{\left(1+t x_{j}+(1-t) y_{j}\right)^{3}}\right)\left(\sum_{j=1}^{m} \frac{t x_{j}+(1-t) y_{j}}{1+t x_{j}+(1-t) y_{j}}\right) \\
& -\left(\sum_{j=1}^{m} \frac{x_{j}-y_{j}}{\left(1+t x_{j}+(1-t) y_{j}\right)^{2}}\right)^{2} \\
\leq & 0,
\end{aligned}
$$

and then $p^{\prime \prime}(t) \leq 0$, that is, $p(t)$ is concave on $[0,1]$.

The proof of Lemma 4 is completed. 
Lemma 5 Let $\mathbf{x}=\left(x_{1}, \ldots, x_{m}\right)$ and $\boldsymbol{y}=\left(y_{1}, \ldots, y_{m}\right) \in\left[\frac{1}{2}, 1\right)^{m}$. Then the function $q(t)=$ $\log \psi(t)$ is convex on $[0,1]$, where

$$
\psi(t)=\sum_{j=1}^{m} \frac{t x_{j}+(1-t) y_{j}}{1-t x_{j}-(1-t) y_{j}}
$$

Proof

$$
q^{\prime}(t)=\frac{\psi^{\prime}(t)}{\psi(t)}
$$

where

$$
\begin{aligned}
\psi^{\prime}(t) & =\sum_{j=1}^{m} \frac{x_{j}-y_{j}}{\left(1-t x_{j}-(1-t) y_{j}\right)^{2}}, \\
q^{\prime \prime}(t) & =\frac{\psi^{\prime \prime}(t) \psi(t)-\left(\psi^{\prime}(t)\right)^{2}}{\psi^{2}(t)},
\end{aligned}
$$

where

$$
\psi^{\prime \prime}(t)=\sum_{j=1}^{m} \frac{2\left(x_{j}-y_{j}\right)^{2}}{\left(1-t x_{j}-(1-t) y_{j}\right)^{3}} .
$$

By the Cauchy inequality, we have

$$
\begin{aligned}
& \psi^{\prime \prime}(t) \psi(t)-\left(\psi^{\prime}(t)\right)^{2} \\
& =\left(\sum_{j=1}^{m} \frac{2\left(x_{j}-y_{j}\right)^{2}}{\left(1-t x_{j}-(1-t) y_{j}\right)^{3}}\right)\left(\sum_{j=1}^{m} \frac{t x_{j}+(1-t) y_{j}}{1-t x_{j}-(1-t) y_{j}}\right)-\left(\sum_{j=1}^{m} \frac{x_{j}-y_{j}}{\left(1-t x_{j}-(1-t) y_{j}\right)^{2}}\right)^{2} \\
& \geq\left(\sum_{j=1}^{m} \frac{\sqrt{2}\left|x_{j}-y_{j}\right|}{\left(1-t x_{j}-(1-t) y_{j}\right)^{\frac{3}{2}}} \frac{\sqrt{t x_{j}+(1-t) y_{j}}}{\sqrt{1-t x_{j}-(1-t) y_{j}}}\right)^{2}-\left(\sum_{j=1}^{m} \frac{x_{j}-y_{j}}{\left(1-t x_{j}-(1-t) y_{j}\right)^{2}}\right)^{2} \\
& =\left(\sum_{j=1}^{m} \frac{\sqrt{2}\left|x_{j}-y_{j}\right| \sqrt{t x_{j}+(1-t) y_{j}}}{\left(1-t x_{j}-(1-t) y_{j}\right)^{2}}\right)^{2}-\left(\sum_{j=1}^{m} \frac{x_{j}-y_{j}}{\left(1-t x_{j}-(1-t) y_{j}\right)^{2}}\right)^{2} .
\end{aligned}
$$

From $x_{j}, y_{j} \in\left[\frac{1}{2}, 1\right)$ it follows that $\sqrt{2} \sqrt{t x_{j}+(1-t) y_{j}} \geq 1$, hence $\psi^{\prime \prime}(t) \psi(t)-\left(\psi^{\prime}(t)\right)^{2} \geq 0$, and then $q^{\prime \prime}(t) \geq 0$, that is, $q(t)$ is convex on $[0,1]$.

The proof of Lemma 5 is completed.

Lemma 6 Let $\mathbf{x}=\left(x_{1}, \ldots, x_{m}\right)$ and $\boldsymbol{y}=\left(y_{1}, \ldots, y_{m}\right) \in\left(0, \frac{1}{2}\right]^{m}$. Then the function $r(t)=$ $\log \varphi(t)$ is convex on $[0,1]$, where

$$
\varphi(t)=\sum_{j=1}^{m} \frac{1-t x_{j}-(1-t) y_{j}}{t x_{j}+(1-t) y_{j}} .
$$

Proof

$$
r^{\prime}(t)=\frac{\varphi^{\prime}(t)}{\varphi(t)},
$$


where

$$
\begin{aligned}
& \varphi^{\prime}(t)=-\sum_{j=1}^{m} \frac{x_{j}-y_{j}}{\left(t x_{j}+(1-t) y_{j}\right)^{2}}, \\
& r^{\prime \prime}(t)=\frac{\varphi^{\prime \prime}(t) \varphi(t)-\left(\varphi^{\prime}(t)\right)^{2}}{\varphi^{2}(t)},
\end{aligned}
$$

where

$$
\varphi^{\prime \prime}(t)=\sum_{j=1}^{m} \frac{2\left(x_{j}-y_{j}\right)^{2}}{\left(t x_{j}+(1-t) y_{j}\right)^{3}} .
$$

By the Cauchy inequality, we have

$$
\begin{aligned}
\varphi^{\prime \prime} & (t) \varphi(t)-\left(\varphi^{\prime}(t)\right)^{2} \\
& =\left(\sum_{j=1}^{m} \frac{2\left(x_{j}-y_{j}\right)^{2}}{\left(t x_{j}+(1-t) y_{j}\right)^{3}}\right)\left(\sum_{j=1}^{m} \frac{1-t x_{j}-(1-t) y_{j}}{t x_{j}+(1-t) y_{j}}\right)-\left(-\sum_{j=1}^{m} \frac{x_{j}-y_{j}}{\left(t x_{j}+(1-t) y_{j}\right)^{2}}\right)^{2} \\
& \geq\left(\sum_{j=1}^{m} \frac{\sqrt{2}\left|x_{j}-y_{j}\right|}{\left(t x_{j}+(1-t) y_{j}\right)^{\frac{3}{2}}} \frac{\sqrt{1-t x_{j}-(1-t) y_{j}}}{\sqrt{t x_{j}+(1-t) y_{j}}}\right)^{2}-\left(\sum_{j=1}^{m} \frac{x_{j}-y_{j}}{\left(t x_{j}+(1-t) y_{j}\right)^{2}}\right)^{2} \\
& =\left(\sum_{j=1}^{m} \frac{\sqrt{2}\left|x_{j}-y_{j}\right| \sqrt{1-t x_{j}-(1-t) y_{j}}}{\left(t x_{j}+(1-t) y_{j}\right)^{2}}\right)^{2}-\left(\sum_{j=1}^{m} \frac{x_{j}-y_{j}}{\left(t x_{j}+(1-t) y_{j}\right)^{2}}\right)^{2} .
\end{aligned}
$$

From $x_{j}, y_{j} \in\left(0, \frac{1}{2}\right]$ it follows that $\sqrt{2} \sqrt{1-t x_{j}-(1-t) y_{j}} \geq 1$, hence $\varphi^{\prime \prime}(t) \varphi(t)-\left(\varphi^{\prime}(t)\right)^{2} \geq 0$, and then $r^{\prime \prime}(t) \geq 0$, that is, $r(t)$ is convex on $[0,1]$.

The proof of Lemma 6 is completed.

Lemma 7 Let $\mathbf{x}=\left(x_{1}, \ldots, x_{m}\right)$ and $\boldsymbol{y}=\left(y_{1}, \ldots, y_{m}\right) \in(0,1)^{m}$. Then the function $h(t)=\log f(t)$ is convex on $[0,1]$, where

$$
f(t)=\sum_{j=1}^{m} \frac{1+t x_{j}+(1-t) y_{j}}{1-t x_{j}-(1-t) y_{j}}
$$

Proof

$$
h^{\prime}(t)=\frac{f^{\prime}(t)}{f(t)},
$$

where

$$
\begin{aligned}
f^{\prime}(t) & =\sum_{j=1}^{m} \frac{2\left(x_{j}-y_{j}\right)}{\left(1-t x_{j}-(1-t) y_{j}\right)^{2}}, \\
h^{\prime \prime}(t) & =\frac{f^{\prime \prime}(t) f(t)-\left(f^{\prime}(t)\right)^{2}}{f^{2}(t)}
\end{aligned}
$$


where

$$
f^{\prime \prime}(t)=\sum_{j=1}^{m} \frac{4\left(x_{j}-y_{j}\right)^{2}}{\left(1-t x_{j}-(1-t) y_{j}\right)^{3}}
$$

By the Cauchy inequality, we have

$$
\begin{aligned}
f^{\prime \prime}(t) f(t)-\left(f^{\prime}(t)\right)^{2} \\
=\left(\sum_{j=1}^{m} \frac{4\left(x_{j}-y_{j}\right)^{2}}{\left(1-t x_{j}-(1-t) y_{j}\right)^{3}}\right)\left(\sum_{j=1}^{m} \frac{1+t x_{j}+(1-t) y_{j}}{1-t x_{j}-(1-t) y_{j}}\right) \\
\quad-\left(\sum_{j=1}^{m} \frac{2\left(x_{j}-y_{j}\right)}{\left(1-t x_{j}-(1-t) y_{j}\right)^{2}}\right)^{2} \\
\geq\left(\sum_{j=1}^{m} \frac{2\left|x_{j}-y_{j}\right|}{\left(1-t x_{j}-(1-t) y_{j}\right)^{\frac{3}{2}}} \frac{\sqrt{1+t x_{j}+(1-t) y_{j}}}{\sqrt{1-t x_{j}-(1-t) y_{j}}}\right)^{2}-\left(\sum_{j=1}^{m} \frac{2\left(x_{j}-y_{j}\right)}{\left(1-t x_{j}-(1-t) y_{j}\right)^{2}}\right)^{2} \\
=\left(\sum_{j=1}^{m} \frac{2\left|x_{j}-y_{j}\right| \sqrt{1+t x_{j}+(1-t) y_{j}}}{\left(1-t x_{j}-(1-t) y_{j}\right)^{2}}\right)^{2}-\left(\sum_{j=1}^{m} \frac{2\left(x_{j}-y_{j}\right)}{\left(1-t x_{j}-(1-t) y_{j}\right)^{2}}\right)^{2} .
\end{aligned}
$$

From $x_{j}, y_{j} \in(0,1)$ it follows that $\sqrt{2} \sqrt{1+t x_{j}+(1-t) y_{j}} \geq 1$, hence $f^{\prime \prime}(t) f(t)-\left(f^{\prime}(t)\right)^{2} \geq 0$, and then $h^{\prime \prime}(t) \geq 0$, that is, $h(t)$ is convex on $[0,1]$.

The proof of Lemma 7 is completed.

Lemma 8 Let $\boldsymbol{x}=\left(x_{1}, \ldots, x_{m}\right)$ and $\boldsymbol{y}=\left(y_{1}, \ldots, y_{m}\right) \in(0, \sqrt{\sqrt{5}-2})^{m}$. Then the function $s(t)=$ $\log w(t)$ is convex on $[0,1]$, where

$$
w(t)=\sum_{j=1}^{m}\left(\frac{1}{t x_{j}+(1-t) y_{j}}-\left(t x_{j}+(1-t) y_{j}\right)\right) .
$$

Proof

$$
s^{\prime}(t)=\frac{w^{\prime}(t)}{w(t)}
$$

where

$$
\begin{aligned}
& w^{\prime}(t)=-\sum_{j=1}^{m}\left(x_{j}-y_{j}\right)\left(\frac{1}{\left(t x_{j}+(1-t) y_{j}\right)^{2}}+1\right), \\
& s^{\prime \prime}(t)=\frac{w^{\prime \prime}(t) w(t)-\left(w^{\prime}(t)\right)^{2}}{w^{2}(t)},
\end{aligned}
$$

where

$$
w^{\prime \prime}(t)=\sum_{j=1}^{m} \frac{2\left(x_{j}-y_{j}\right)^{2}}{\left(t x_{j}+(1-t) y_{j}\right)^{3}} .
$$


By the Cauchy inequality, we have

$$
\begin{aligned}
w^{\prime \prime}(t) w(t)-\left(w^{\prime}(t)\right)^{2} & \left(\sum_{j=1}^{m} \frac{2\left(x_{j}-y_{j}\right)^{2}}{\left(t x_{j}+(1-t) y_{j}\right)^{3}}\right)\left(\sum_{j=1}^{m}\left(\frac{1}{t x_{j}+(1-t) y_{j}}-\left(t x_{j}+(1-t) y_{j}\right)\right)\right) \\
& -\left(-\sum_{j=1}^{m}\left(x_{j}-y_{j}\right)\left(\frac{1}{\left(t x_{j}+(1-t) y_{j}\right)^{2}}+1\right)\right)^{2} \\
\geq & \left(\sum_{j=1}^{m} \frac{\sqrt{2}\left|x_{j}-y_{j}\right|}{\left(t x_{j}+(1-t) y_{j}\right)^{\frac{3}{2}}} \sqrt{\left.\frac{1}{t x_{j}+(1-t) y_{j}}-\left(t x_{j}+(1-t) y_{j}\right)\right)^{2}}\right. \\
& -\left(\sum_{j=1}^{m}\left(x_{j}-y_{j}\right)\left(\frac{1}{\left(t x_{j}+(1-t) y_{j}\right)^{2}}+1\right)\right)^{2} \\
= & \left(\sum_{j=1}^{m} \frac{\sqrt{2}\left|x_{j}-y_{j}\right| \sqrt{1-\left(t x_{j}+(1-t) y_{j}\right)^{2}}}{\left(t x_{j}+(1-t) y_{j}\right)^{2}}\right)^{2}-\left(\sum_{j=1}^{m}\left(x_{j}-y_{j}\right) \frac{1+\left(t x_{j}+(1-t) y_{j}\right)^{2}}{\left(t x_{j}+(1-t) y_{j}\right)^{2}}\right)^{2} .
\end{aligned}
$$

Let $u_{j}:=t x_{j}+(1-t) y_{j}$. From $x_{j}, y_{j} \in(0, \sqrt{\sqrt{5}-2})$ it follows that $u_{j}^{2} \leq \sqrt{5}-2$. Since

$$
\begin{gathered}
u_{j}^{2} \leq \sqrt{5}-2 \quad \Leftrightarrow \quad\left(u_{j}^{2}+2\right)^{2} \leq 5 \quad \Leftrightarrow \quad u_{j}^{4}+4 u_{j}^{2}-1 \leq 0 \\
\Leftrightarrow \quad 2\left(1-u_{j}^{2}\right) \geq\left(1+u_{j}^{2}\right)^{2} \quad \Leftrightarrow \quad \sqrt{2} \sqrt{1-u_{j}^{2}} \geq 1+u_{j}^{2},
\end{gathered}
$$

so $w^{\prime \prime}(t) w(t)-\left(w^{\prime}(t)\right)^{2} \geq 0$, and then $s^{\prime \prime}(t) \geq 0$, that is, $s(t)$ is convex on $[0,1]$.

The proof of Lemma 8 is completed.

\section{Proof of main results}

Proof of Theorem 4 For any $1 \leq i_{1}<\cdots<i_{k} \leq n$, by Lemma 3 and Lemma 7 , it follows that $\log \sum_{j=1}^{k} \frac{1+x_{i j}}{1-x_{i j}}$ is convex on $(0,1)^{k}$. Obviously, $\log \sum_{j=1}^{k} \frac{1+x_{i j}}{1-x_{i j}}$ is also convex on $(0,1)^{n}$, and then $\log E_{k}^{*}\left(\frac{1+\boldsymbol{x}}{1-\boldsymbol{x}}\right)=\sum_{1 \leq i_{1}<\cdots<i_{k} \leq n} \log \sum_{j=1}^{k} \frac{1+x_{i_{j}}}{1-x_{i_{j}}}$ is convex on $(0,1)^{n}$. Furthermore, it is clear that $\log E_{k}^{*}\left(\frac{1+\boldsymbol{x}}{1-\boldsymbol{x}}\right)$ is symmetric on $(0,1)^{n}$. By Lemma 1 , it follows that $\log E_{k}^{*}\left(\frac{1+\boldsymbol{x}}{1-\boldsymbol{x}}\right)$ is Schurconvex on $(0,1)^{n}$, and then from Lemma 2 we conclude that $E_{k}^{*}\left(\frac{1+x}{1-x}\right)$ is also Schur-convex on $(0,1)^{n}$.

The proof of Theorem 4 is completed.

Similar to the proof of Theorem 4, we can use Lemma 4, Lemma 5, Lemma 6 and Lemma 8 respectively to prove Theorem 1, Theorem 2, Theorem 3 and Theorem 5 ; therefore we omit the details of the proof.

Remark 1 Using the Schur-convex function decision theorem, Liu et al. [9] have proved Theorem 3. Xia and Chu [10] have proved that the symmetric function

$$
E_{k}^{*}\left(\frac{1+\boldsymbol{x}}{\boldsymbol{x}}\right)=\prod_{1 \leq i_{1}<\cdots<i_{k} \leq n} \sum_{j=1}^{k} \frac{1+x_{i_{j}}}{x_{i_{j}}}, \quad k=1, \ldots, n,
$$

is a Schur-convex function on $\mathbb{R}_{+}^{n}$. 
The reader may wish to prove the inequality (12) by the properties of a Schur-convex function.

\section{Competing interests}

The authors declare that they have no competing interests.

\section{Authors' contributions}

The authors co-authored this paper together. All authors read and approved the final manuscript.

\section{Author details}

'Department of Electronic Information, Teacher's College, Beijing Union University, Beijing, 100011, P.R. China. ${ }^{2}$ Basic Courses Department, Beijing Union University, Beijing, 100101, P.R. China.

\section{Acknowledgements}

The work was supported by Funding Project for Academic Human Resources Development in Institutions of Higher Learning under the Jurisdiction of Beijing Municipality (PHR (IHLB)) (PHR201108407). Thanks for the help.

Received: 16 April 2013 Accepted: 8 June 2013 Published: 17 June 2013

\section{References}

1. Marshall, AW, Olkin, I, Arnold, BC: Inequalities: Theory of Majorization and Its Application, 2nd edn. Springer, New York (2011)

2. Wang, BY: Foundations of Majorization Inequalities. Beijing Normal University Press, Beijing (1990) (in Chinese)

3. Xia, WF, Wang, GD, Chu, YM: Schur convexity and inequalities for a class of symmetric functions. Int. J. Pure Appl. Math. 58(4), 435-452 (2010)

4. Chu, YM, Xia, WF, Zhao, TH: Schur convexity for a class of symmetric functions. Sci. China Math. 53(2), 465-474 (2010)

5. Xia, WF, Chu, YM: Schur convexity and Schur multiplicative convexity for a class of symmetric functions with applications. Ukr. Math. J. 61(10), 1541-1555 (2009)

6. Xia, W-F, Chu, Y-M: On Schur-convexity of some symmetric functions. J. Inequal. Appl. 2010, Article ID 543250 (2010). doi:10.1155/2010/543250

7. Mei, H, Bai, CL, Man, H: Extension of an inequality guess. J. Inn. Mong. Univ. Natl. 21(2), 127-129 (2006) (in Chinese)

8. Shi, H-N: Theory of Majorization and Analytic Inequalities. Harbin Institute of Technology Press, Harbin (2012) (in Chinese)

9. Liu, HQ, Yu, Q, Zhang, Y: Some properties of a class of symmetric functions and its applications. J. Hengyang Norm. Univ. 33(6), 167-171 (2012) (in Chinese)

10. Xia, W, Chu, Y: Schur convexity with respect to a class of symmetric functions and their applications. Bull. Math. Anal. Appl. 3(3), 84-96 (2011). http://www.bmathaa.org

doi:10.1186/1029-242X-2013-295

Cite this article as: Shi and Zhang: Schur-convexity of dual form of some symmetric functions. Journal of Inequalities and Applications 2013 2013:295.

\section{Submit your manuscript to a SpringerOpen ${ }^{\circ}$ journal and benefit from:}

- Convenient online submission

- Rigorous peer review

Immediate publication on acceptance

- Open access: articles freely available online

- High visibility within the field

- Retaining the copyright to your article 